\title{
A Foundation Wall Heat Exchanger Model and Validation Study
}

\author{
I da Shafagh
}

\begin{abstract}
$\mathrm{M}$ aking use of foundation substructural elements as ground heat ex changers is an attractive option for larger non-residential buildings. A $\mathrm{n}$ alternative to E nergy Piles is to use wall substructures - so called diaphragm or screen walls - with embedded pipes that are partly below ground and partly ex posed to basement spaces. This paper will describe the development of a model of such a heat ex changer that uses a weighting factor approach $\mathrm{known}$ as $\mathrm{D}$ ynamic Thermal N etwork (D T N). This approach allows for detailed representation of the wall section geometry and multiple boundary onditions. In this case thermal boundary onditions are applied at surfaces representing the adjacent ground and the semiex posed basement wall surface in addition to the pipe surface. The weighting factors for the model have been derived using a parametric numerical model that has been developed using the 0 penF OA M library. V alidation of the model has been carried out using data from an ex tended series of thermal response test (TRT) measurements at a full-scale diaphragm wall heat ex changer in Barcelona. In this paper, development of the model using the D TN approach will be briefly described along with the parametric numerical modelling approach used to derive the weighting factor data. $\mathrm{V}$ alidation test prooedures will be presented along with comparisons between the predicted and measured fluid temperatures and heat transfer rates. G iven some uncertainty in the ex perimental thermal properties, the model was able to predict the dynamics of thermal response over a range of operating conditions with reasonable acouracy and using very modest computational resouroes.
\end{abstract}

\section{NTRODUCTI ON}

One of the grand challenges confronting the world today is to meet the growing demand for energy while addressing the environmental and climate impacts of fossil fuels consumptions. A large portion of energy consumptions in developed countries corresponds to heating of residential and commercial buildings (Pérez-Lombard et al., 2008). Exploitations of sustainable and renewable energy resources for direct use as thermal energy in such spaces can significantly reduce the need for conventional fuels and therefore decrease greenhouse gas emissions. To that end, geothermal energy represents an indispensable choice for its applicability in variable atmospheric conditions. Depending on its application geothermal energy can be extracted from deep or near surface reservoirs. Shallow geothermal energy is mainly used for the purpose of heating and cooling of buildings, referred to as its direct use. One important aspect of direct geothermal use is its applicability in almost all geographic locations (Lund et al., 2011).

To extract energy from the ground reinforced substructural elements equipped with heat exchanger pipes are used (Florides and Kalogimu, 2007). The ground works well as a heat exchanger since the underground temperature stays constant throughout the year and the ground temperature below $10 \mathrm{~m}$ underground is not affected by the seasonal changes in outdoor air temperature (D roulia et al., 2009). Using substructures as heat exhchangers started in 
the 1980s, starting from ground-bearing slabs with the first adoption of diaphragm walls as heat exchangers being reported in 1996 in Austria and Switzerland (Brandl, 2006).

To date, numerious investigations into performance of thermos-active piles have been carried out (Esen and Inalli, 2009; Hepbasli et al., 2003; Li et al., 2006; Lim et al., 2007) while diaphragm wall heat exchangers (DWHE) are less researched (Rees, 2016). Brandl (2006) studied heat transfer in diaphragm walls applied in three main pilot projects including a rehabilitation centre, a traffic tunnel, and metro stations in addition to further smaller projects. Adam and Markiewicz (2009) performed finite element simulations to calculate heating and cooling performance of absorber elements such as diaphragm walls at different absorber distances. They reported that with larger distances the thermal power will decrease as well as the installation cost tending to an optimum. However, it was emphasized that their results are only valid for the studied case and may vary significantly for other geothermal systems while the method is applicable to any geothermal energy system installed in foundation elements. Xia et al. (2013) made the first attempt to experimentally investigate heat transfer performance of DWHEs and through comparison with borehole heat exchangers (BHE) revealed heat transfer charactenistics of DWHEs and the factors influencing heat exchange rate in such geostructures. Sun et al. (2013) developed two-dimensional heat transfer models for DWHEs according to the structural features, i.e. over and under the excavation line, based on which a design model for such energy geostructures was proposed. Their models showed good agreement with numerical solutions and measured data.

Kürten et al. (2013) proposed a new numerical approach for the thermal analysis of DWHEs and verified their findings with laboratory tests. Boume et al. (2016b) conducted numerical analysis to establish the heat exchange mechanisms in DWHEs, and reported that the main mechanism is between the air-void and wall rather than the ground. In another study Boume-Webb et al. (2016a) identified communalities and differences between the methods used for evaluating BHEs and energy geostructures. Sterpi et al. (2017) investigated the energy performance and short and long term influence on the soil temperatures using finite element theml analysis. Furthermore, they carried out finite element thermo-mechnical analysis to highlight the wall geothecnical and structural response. Coletto and Sterpi (2016) used coupled thermo-mechanical analysis to study the heat transfer effects on the soil temperatures, the wall internal actions and the soil-structure interaction. Di Donna et al. (2016) used numerical simulation and statistical analysis to highlight the parameters governing energy efficiency in DWHEs. Soga and Rui (2016) summerised the current understanding on the performance of energy geostructures and discussed some design considerations. They suggested that more work is required to build confidence in the use of such substructure heat exchangers.

Major insights into heat transfer processes between DWHEs and their surrounding boundaries can be provided by monitoring temperature data from full-scale in-situ cases which conventionally is carried out using a thermal response test (TRT) for substructure heat exchangers. In the present work, a TRT apparatus that injects heat energy at a constant rate into one end of the loop and measures the outflow temperature at the other end is used to stimulate the heat exchanger and derive data for model validation. In contrast to a TRT we are not seeking to parametrically evaluate the ground or concrete thermal properties. To interprete such temperature data and evaluate temperature evolution in the circulating fluid as a function of time a suitable heat transfer mathematical model is required. Various mathematical models are inrtroduced for the analysis of heat exchange processes whithin a borehole heat exchanger and pile foundations, however, there are few models concerning DWHE due to their complexity. Temperature profiles predicated by the two-dimensional heat transfer model developed by Sun et al. (2013) show limited consistency with the experimental data but have been considered adequate by the authors. A thermal resistance model that takes rotational symmetry, number of pipes, and the spatial separation into account was presented and implemented into a finite difference code by Kurten et al. (2015). Their model does not consider seasonal fluctuation of the near surface temperature and the groundwater. In the present work, a combination of finite volume analysis and an approach known as dynamic thermal networks (DTN) proposed by Claesson (2002) and Wentzel (2005) is used for calculating dynamic conduction heat transfer in D WHEs. DTN is a response factor method that can provide efficient simulation of complex three-dimensional geometries such as DWHE with the basement, pipe and adjacent ground as temperature boundaries. To that end, the comesponding geometry of a D WHE is created and dicretized in 
OpenFOAM (Weller et al., 1998). O penFOAM was also employed to obtain the weighting factor series required as inputs to the DTN model. The experimental inlet temperature, ambient temperature, and the thermal properties of the ground and concrete were employed in the model to predict the outlet temperature of the DWHE over a period of 8 weeks. The method is verified against experimental data collected from an in-situ DWHE installation.

\section{APHRAGM WALL HEAT EXCHANGER (DWHE)}

A schematic representation of a diaphragm wall equipped with heat exchanger pipes is illustrated in Figure 1-a and Figure 1-b. The wall depth varies depending on its application. High density polyethylene pipe (HD PE) or cross-linked polyethene (PEX) pipe is typically used to contain the heat exchange fluid in a closed loop. The pipes are installed by attachement to the reinforcement steel cage of the concrete wall closer to the side facing the ground. There are various possible layouts for arranging the pipe whithin the wall; the one shown in Figure 1 is a common configuration. Walls are typically constructed in panels depending on the practicalities of assembly and lifting and it is often convenient to arrange pipe circuits to be divided accordingly, with some consideration to available pipe lengths and limiting hycraulic pressure drops.

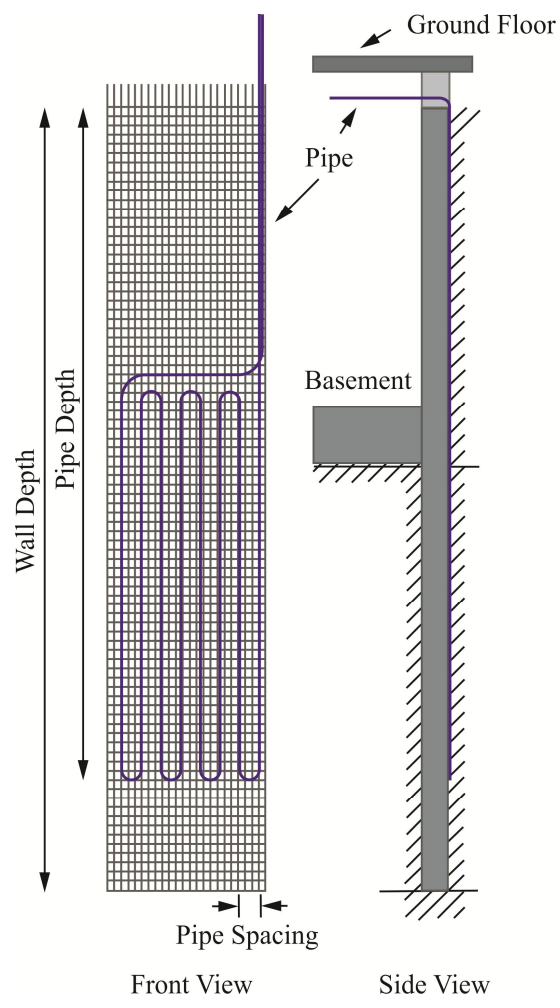

(a)

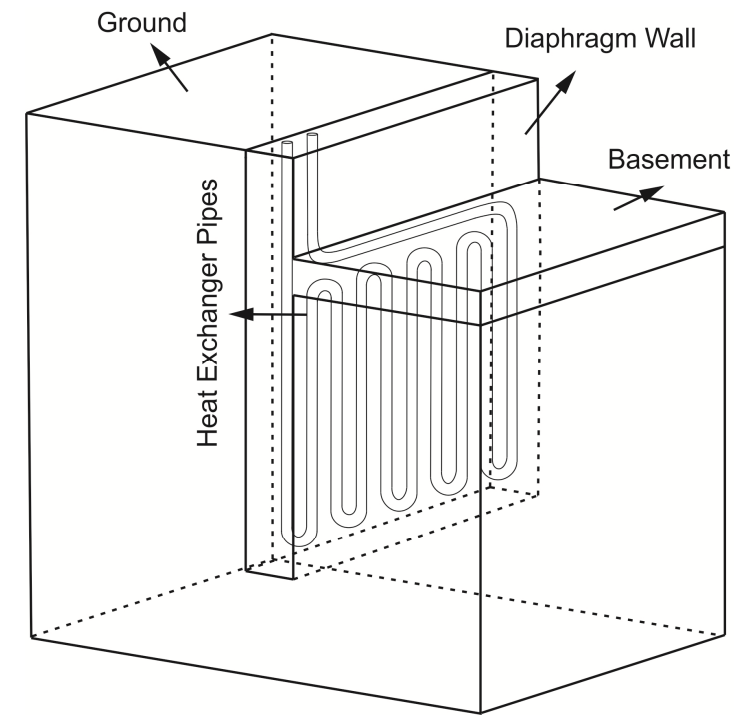

(b)

Figure 1 Schematic representation of a diaphragm wall heat exchanger and its surrounding boundaries.

\section{HEAT TRANSFER MODEL}

The DWHE studied here consists of a diaphragm wall of $17 \mathrm{~m}$ depth and thickness of $0.6 \mathrm{~m}$ embedded with a $93 \mathrm{~m}$ long pipe cuircuit looped in veritical orientation (4 loops as shown in Figure 1). Pipe inner and outer diameters 
are $21 \mathrm{~mm}$ and $25 \mathrm{~mm}$, respectively. To simplify the calculations and considering the symmetric configuration of the wall an isolated section containing only one pipe is considered. The corresponding geometry was created and dicretized in OpenFOAM (Weller et al., 1998) using the respective blockMesh utility, as shown in Figure 2. This parametric mesh generation tool allows generation of meshes in an automatied manner. An additional utility was developed to allow generation of D WHE meshes from relatively few design parameters.

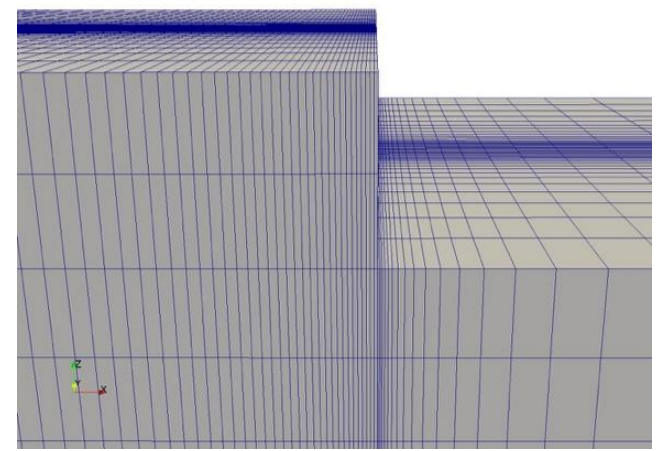

(a)

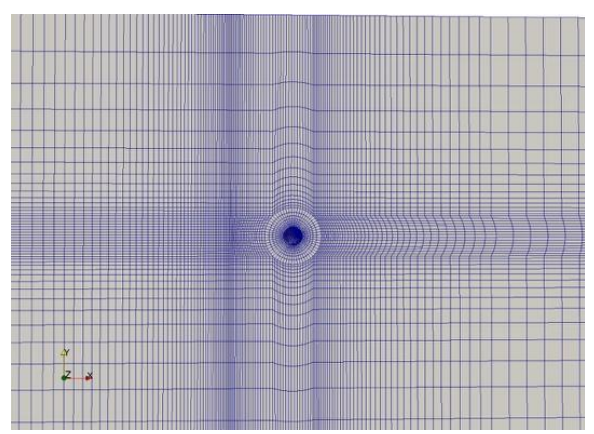

(b)

Figure 2 The validation model numerical mesh showing the top of the wall (a) and the pipe (b).

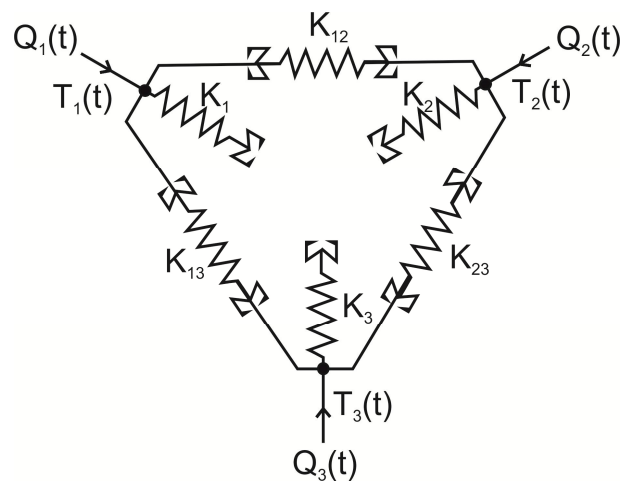

(a)

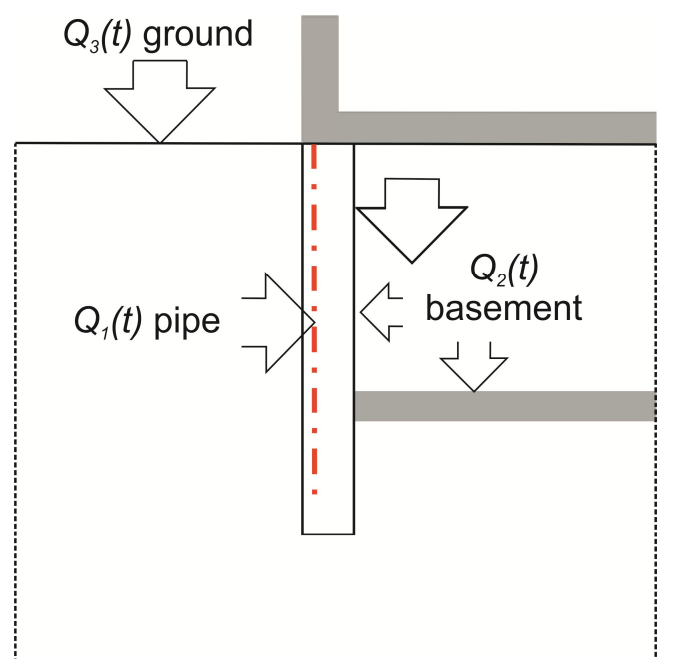

(b)

Figure 3 A threesurface form of Dynamic Thermal Network showing the resistance and nodes (a) and the corresponding surfaces in a DTN representation of a D WHE problem (b).

For DTN calculations, the time-dependent thermal processes are represented as a network to describe the relationship between boundary temperatures and heat fluxes. This network includes a combination of admittive and transmittive heat paths and time-varying conductances that are characterized by a series of response factors (Figure 
3-a). The method can be shown to be exact in both continuous and discrete forms and can be applied, in principle, to arbitrary geometries with heterogeneous thermal properties (Claesson, 2002; Rees and Fan, 2013; Wentzel, 2005). This makes the method very attractive for energy pile and DWHE problems. In such situations we are interested in specifying complex geometries with two sets of thermal properties (concrete and ground) and representing three boundary condtions: (1) the pipe surfaces, (2) the basement surface and (3) the upper gorund surface. The fluxes at these boundaries are denoted $Q_{1}, Q_{2}$ and $Q_{3}$ respectively as shown in Fig.3.

An essential feature of the DTN method is that fluxes at each surface are separated into transmittive and absorptive components. So that, using node 1 as an example, the transient heat balance equation is expressed as:

$$
Q_{1}(t)=Q_{1 a}(t)+Q_{12}(t)+Q_{13}(t)
$$

where $Q_{1 a}$ is the absorbtive flux and $Q_{12}$ and $Q_{13}$ are the transmittive fluxes. Rather than these fluxes being functions of instantaneous node temperatures and conductances as in a steady-state network representation, the transient fluxes are experessed as functions of constant conductances and weighted average node temperatures so that for node 1:

$$
Q_{1}(t)=K_{1} \cdot\left[T_{1}(t)-\bar{T}_{1 a}(t)\right]+K_{12} \cdot\left[\bar{T}_{12}(t)-\bar{T}_{21}(t)\right]+K_{13} \cdot\left[\bar{T}_{13}(t)-\bar{T}_{31}(t)\right]
$$

where $T_{1}(t)$ is the current boundary temperature and $\bar{T}_{1 a}(t)$ is the weighted average absorbtive temperature and $\bar{T}_{12}(t)$ and $\bar{T}_{13}(t)$ are the weighted mean transmittive temperatures. In discrete form these temperatures are expressed as summations of past temperatures multiplied by respective weighting factors as follows:

$$
\bar{T}_{i a, n}=\sum_{\rho=1}^{\infty} \kappa_{i a, \rho} \cdot T_{i, n-\rho} \bar{T}_{i j, n}=\sum_{\rho=0}^{\infty} \kappa_{i j, \rho} \cdot T_{i, n-\rho}
$$

where $\kappa_{i a, \rho}$ is an absorptive weighting factor and $\kappa_{i j, \rho}$ is a discrete transmittive weighting factor in a finite series of such weighting factors. These weighted average temperatures are updated from one timestep to the next. This gives a heat balance equation for boundary 1 of the problem in the following form:

$Q_{1, n}=\bar{K}_{1} \cdot\left[T_{1, n}-\sum_{\rho=1}^{\rho_{s}} \kappa_{1 a, \rho} T_{1, n-\rho}\right]+K_{12} \cdot \sum_{\rho=0}^{\rho_{s}} \kappa_{12, \rho}\left(T_{1, n-\rho}-T_{2, n-\rho}\right)+K_{13} \cdot \sum_{\rho=0}^{\rho_{s}} \kappa_{13, \rho}\left(T_{1, n-\rho}-T_{3, n-\rho}\right)$

This equation is a computationally cheap summation of temperatures multiplied by weighting factors and so the model is very efficient. Some effort is required in deriving the sets of weighting factors, however, these can be conveniently derived by applying step-changes in temperatures at each boundary in tum. In our case we use the O penFOAM numerical model described above to do this. The time-series fluxes from these calculations are then used to derive the weighting factors. D etails of the process are desicribed in Wentzel (2005) and Rees and Fan (2013) along with detailed descriptions of the boundary condtion treatment for the pipe and exposed surfaces. 


\section{RESULTS AND DISCUSSIONS}

Model validation has been attempted by making comparisons with experimental DWHE data over a period of 6 weeks and the results are displayed in Figure 4. The experimental testing program was conducted during the construction of a demonstration building in Barcelona. A series of heat rejection pulses were applied using thermal response test (TRT) equipment. In these experiments the inlet and outet fluid temperatures into and from the diaphragm wall were measured at $5 \mathrm{~min}$ intervals from $18^{\text {th }}$ September to 30th 0 ctober 2017. D uring the experiments the heat pump was switched on and off intermittently while the circulating pump ran continuously. Figure 4-a to Figure 4-c show the profile of the inlet, outlet, and ambient temperatures measured during the test as well as measured heat transfer rate. Predicated outlet temperature and heat trasnefr rate using the DTN DWHE model are also pesented. The wall dimensions and properties are detailed in Table 1. Three stages of measurements can be identified during this test series as shown in Figure 4. During the first stage, the pump ran for relatively longer hours before being switched off. The length of the cycles was reduced in stage two and are shorter again (2 hours) in stage three.

It was not possible to obtain independent measurements of concrete and ground thermal properties at the site. Consequently, we have investigated a range of property values (somewhat heuristically) to investigate the sensitivity of the model results. Accordingly, calculations using the DTN diaphragm wall model have been performed using thermal conductivity values ranging from $1.8-3.0 \mathrm{Wm}^{-1} \mathrm{~K}^{-1}$ for concrete and $0.6-2.0 \mathrm{Wm}^{-1} \mathrm{~K}^{-1}$ for the ground. In addition, volumetric heat capacity values of $1.6 \times 10^{6}-3.75 \times 10^{6} \mathrm{~J} \mathrm{~m}^{-3} \mathrm{~K}^{-1}$ are examined for concrete while one single value of $1.6 \times 10^{6} \mathrm{~J} \mathrm{~m}^{-3} \mathrm{~K}^{-1}$ is used for ground. The results indicated that there is sensitivity to both the concrete and the ground themal conductivities. D ata from the shortest cycle periods was used to guide the choice of concrete properties as heat transfer variations are mostly limited to within the concrete in such conditions. Conversely, data from the longer cycles of heat rejection were more sensitive to ground thermal properties.

Table 1. DTN DWHE Model Parameters for the TRT Test Conditions

\begin{tabular}{lll}
\hline \multicolumn{1}{c}{ Model Parameters } & \multicolumn{1}{c}{ Value } & Units \\
\hline \hline Wall D epth & 17.0 & $\mathrm{~m}$ \\
Pipe D epth & 15.6 & $\mathrm{~m}$ \\
Basement D epth & 6.5 & $\mathrm{~m}$ \\
Pipe outer diameter & 25 & $\mathrm{~mm}$ \\
Pipe inner diameter & 21 & $\mathrm{~mm}$ \\
Pipe horizontal spacing & 0.40 & $\mathrm{~m}$ \\
Pipe circuit length & 93.0 & $\mathrm{~m}$ \\
Number of loops & 4 & - \\
Pipe thermal conductivity & 0.39 & $\mathrm{~W} \mathrm{~m}-1 \mathrm{~K}^{-1}$ \\
Fluid conductivity & 0.625 & $\mathrm{~W} \mathrm{~m} \mathrm{~K}^{-1}$ \\
Fluid specific heat & 4178 & $\mathrm{~J} \mathrm{kg-1} \mathrm{K}^{-1}$ \\
Fluid density & 994.0 & $\mathrm{~kg} \mathrm{~m}^{-3}$ \\
Fluid viscosity & 0.000714 & $\mathrm{~Pa}$ \\
\hline
\end{tabular}

Table 2. Thermal Properties of Concrete and Ground Used in Figure 2

\begin{tabular}{lll}
\hline \multicolumn{1}{c}{ Themal Propenties } & Value & Units \\
\hline \hline Concrete thermal conductivity & 2.25 & $\mathrm{~W} \mathrm{~m}^{-1} \mathrm{~K}^{-1}$ \\
Ground thermal conductivity & 1.6 & $\mathrm{~W} \mathrm{~m}^{-1} \mathrm{~K}^{-1}$ \\
Concrete volumetric heat capacity & $3.5 \times 10^{6}$ & $\mathrm{~J} \mathrm{~m}^{-3} \mathrm{~K}^{-1}$ \\
Ground volumetric heat capacity & $1.6 \times 10^{6}$ & $\mathrm{~J} \mathrm{~m}^{-3} \mathrm{~K}^{-1}$ \\
\hline
\end{tabular}



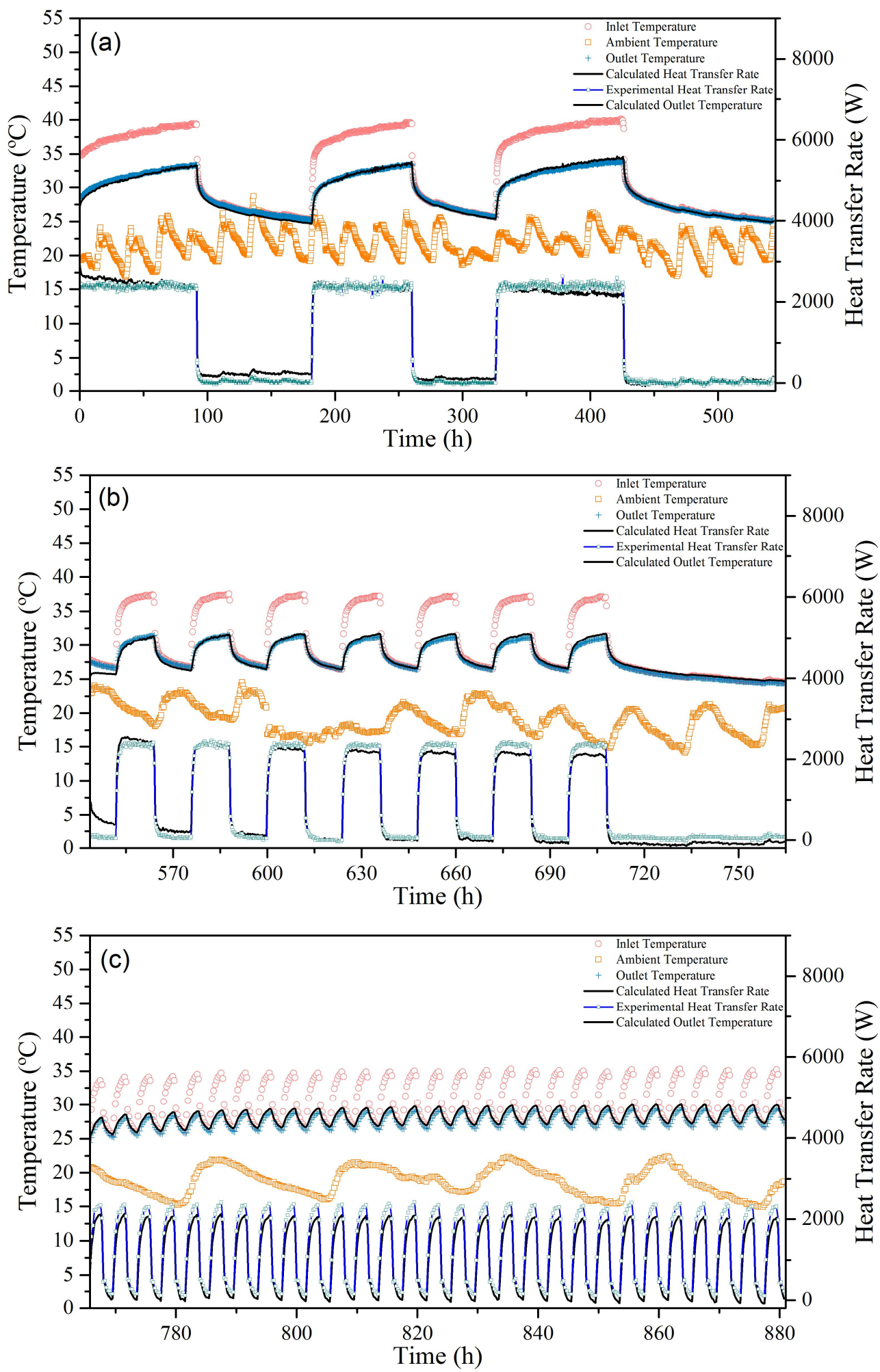

Figure 4 Hourly variations in measured temperatures and heat transfer rate compared with calculated data. 
Calculated time series in Figure 4 represents the best fit with the experiments for which comesponding thermal properties of ground and concrete are shown in Table 2. Predicted outlet temperature and heat trasnefr rate follow the experiments closely over the operation period. The root mean square error (RMSE) between the calculated and measured outlet temperatures over the 6 weeks operation period is $0.4 \mathrm{~K}$ which represents a good level of agreement for modelling purposes. Data in Table 2 indicates that the closest agreement is found with higher values of ground and concrete thermal conductivities and relatively high value of concrete volumetric heat capacity. We believe using values that are higher than that for plain concrete are justifyable in view of the significant level of reinforcement steel surrounding the pipes. In this model the wall thermal properties are effective or composite values for the wall material. The issue of the impact of the reinforcement on the nature of short timescale response is worthy of further investigation.

The validity of the DTN DWHE analogy to define heat transfer at the wall in the proposed model has been investigated by examining prediction of ground heat transfer over the operation period. The model compares favourably with the experimental data. The measured heat rejection over the whole period is $999.7 \mathrm{kWh}$ and this compares with a predicted value of $988.7 \mathrm{kWh}$ which corresponds to a $1.10 \%$ relative error and this seems an acceptable value. Completing calculations for the whole experimental data series required of the order of one minute of computing time.

\section{CONCLUSIONS}

A heat transfer model has been proposed that combines a numerical finite volume representation of a diaphragm wall heat exchanger and surrounding ground and basement boundaries and a Dynamic Thermal Network (DTN) representation derived from the numerical data. The model validation testing has been carried out using a themal response test (TRT) approach over an extended period with different periods of cyclic operation. It has been shown that the results are sensitive to thermal property values of the ground and concrete. Values of effective thermal capacity were chosen at the upper end of the usual range and this seems justifyable in view of the large amount of reinforcement steel in the wall. The relative errors in outlet temperature between the DTN model and the measured data are no more than $0.4 \mathrm{~K}$ for an operation period of about $880 \mathrm{~h}$. The levels of agreement in predicted dynamic performance are concluded to be more than satisfactory for heat exchanger design and TRT analysis purposes. The model is relatively efficient and so well suited to analysis of long-term performance analysis.

\section{ACKNOWLEDGMENTS}

This work was possible thanks to the research project $\mathrm{H} 2020 \mathrm{GEOTeCH}$. GEOTeCH is co-funded by the European Community Horizon 2020 Program for European Research and Technological Development (2014-2020) and has received research funding from the European Union (www.geotech-project.eu) under grant agreement No. 656889. Experimental data was kindly provided by project partners Technalia, COMSA, and ARC BCN.

\section{REFERENCES}

Adam, D. and Markiewicz, R. 2009. E nergy from E arth-Coupled Structures, Foundations, Tunnels and Sewers. Géotechnique. 59(3), pp.229-236.

Bourne-Webb, P. et al. 2016a. A nalysis and D esign M ethods for E nergy G eostructures. Renewable and Sustainable Energy Reviews. 65, pp.402-419.

Bourne-Webb, P.J. et al. 2016b. Thermal and M echanical A spects of the Response of E mbedded Retaining W alls U sed as Shallow G eothermal $\mathrm{H}$ eat $\mathrm{E} x$ changers. Energy and Buildings. 125, pp.130-141.

Brandl, H. 2006. E nergy Foundations and 0 ther Thermo-A ctive G round Structures. Géotechnique. 56(2), pp.81-122.

Claesson, J. 2002. D ynamic Thermal Networks. Outlines of A General Theory. In: Proceedings of the 6th Symposium on Building Physics in the Nordic Countries, Trondheim, Norway. pp.47-54.

Coletto, A. and Sterpi, D. 2016. Structural and G eotechnical E ffects of Thermal L oads in E nergy W alls. Procedia Engineering. 158, pp.224229. 
Di D onna, A. et al. 2016. E nergy Performance of D iaphragm W alls U sed as H eat E x changers. Geotechnical Engineering. 170(GE3), pp.232-245.

Kürten, S. et al. 2015. A new model for the description of the heat transfer for plane thermo-active geotechnical systems based on thermal resistanoes. Acta G eotechnica. 10(2), pp.219-229.

Rees, S.J. and Fan, D. 2013. A N umerical Implementation of the D ynamic Thermal N etwork Method for L ong Time Series Simulation of Conduction in M ulti-dimensional N on-homogeneous Solids. International Journal of Heat and Mass Transfer. 61, pp.475-489.

Soga, K. and Rui, Y. 2016. 7 - E nergy geostructures A 2 - Rees, Simon J. Advances in G round-Source Heat Pump Systems. Woodhead Publishing, pp.185-221.

Sterpi, D. et al. 2017. Investigation on the Behaviour of a Thermo-A dive D iaphragm W all by Thermo-M echanical A nalyses. Geomechanics for Energy and the Environment. 9, pp.1-20.

Sun, M. et al. 2013. Heat Transfer Model and D esign Method for G eothermal $\mathrm{H}$ eat Exchange Tubes in D iaphragm W alls. Energy and Buildings. 61, pp.250-259.

Weller, H.G . et al. 1998. A Tensorial A pproach to Computational C ontinuum Mechanics U sing 0 bject-oriented Techniques. Computers in Physics. 12(6), pp.620-631.

Wentzel, E.-L. 2005. Thermal Modeling of W alls, Foundations and W hole Buildings U sing D ynamic Thermal N etworks. thesis, Chalmers University of Technology. 